\title{
TEXTILE RESEARCH IN AUSTRALIA
}

\author{
By Dr. M. LIPSON \\ Chief, C.S.I.R.O. Division of Textile Industry, Wool Research Laboratories, \\ Geelong, Victoria
}

$\mathrm{O}^{\mathrm{N}}$ May 3 the Prime Minister of the Commonwealth of Australia, Sir Robert Menzies, officially opened a new laboratory block at the C.S.I.R.O. Division of Textile Industry, Geelong (Fig. 1). A large gathering was present from many spheres, including parliamentary and civic representatives, as well as representatives of the wool industry, scientific research and teaching organizations. The new building has an approximate area of 50,000 $\mathrm{ft} .^{2}$. It consists of a two-storey, air-conditioned block to house physical and chemical research laboratories, general offices and library linked to a single-storey structure used for amenities and containing a conference hall. A large counterbalanced wall separating the conference hall from the amenities area can be lowered into the ground to provide an area seating up to 1,000 when required for large meetings.

Research at the Division in the past thirteen years has been mainly concerned with major problems and improvements in wool textile processing and the development of new and better consumer products from wool. In addition to normal laboratory facilities, there is an experimental wool textile mill with all stages of processing from greasy fleece to finished fabric. A large, well-equipped workshop ensures that laboratory findings can be translated to the full scale with a minimum of delay. Some of the research has led to methods which are now widely used in the wool textile industry. Probably the most widely applied of these is a technique for moth-proofing wool by application of dieldrin from emulsion during dyeing or finishing. It was found that dieldrin, applied to wool in this way, had unexpected fastness to dry cleaning or washing which did not occur with application from organic solvents. In Australia something like 90 per cent of wool processed in local mills is now moth-proofed in this way and the technique has extended to Britain, the United States, Japan and Continental countries.

Another development is a method for overcoming felting shrinkage in wool based on treatment with potassium permanganate in saturated sodium chloride solution. By inhibiting fibre swelling, the saturated salt solution restricts action of the permanganate to the surface scales of the fibres. Apart from shrink-proofing wool by means of scale degradation, other methods, such as resin deposi- tion, have been closely studied. It has been found that traces of impurities in the fibre which remain after normal wool scouring can inhibit the shrink-proofing action of resins. When a fabric is extracted with alcohol, a subsequent resin treatment is usually much more effective. Investigations are in progress to ascertain the nature and location of these impurities using infra-red and chromatographic techniques. Such work could open the way to more effective treatments with smaller quantities of resins. Another line of research has been in analysing the scales removed from fibres before and after treatment with various reagents used in shrink-proofing wool by surface modification. So far, this work has shown that the only major chemical change in the fibre is reaction with cystine, thus supporting the views of Speakman and co-workers.

A method for permanent pleating and creasing wool fabrics was developed some years ago in which the wool is sprayed with a solution of ammonium thioglycollate just prior to pressing the final garment. Other reducing agents have been found equally effective. This work was developed in conjunction with more fundamental studies on the setting properties of wool fibres. More recent research is concerned with developing a dry-setting technique for permanent pleating and creasing of wool. By this method, the mill should be able to apply the setting reagent to the cloth during finishing and the tailor would be able to press without the addition of any solution or without. wetting the cloth which was not hitherto possible. Of more fundamental interest is research on the sorption by wool of organic liquids, such as alcohols. Marked and unexpected changes have resulted from modification of certain of the polar side-chains.

Research on textile processing has been concerned mainly with the earlier stages of manufacture involved in going from greasy wool to the top which requires processes peculiar to wool, such as scouring, carding and combing. A method for solvent degreasing of wool has been developed in place of conventional scouring. This technique has been commercially applied in Australia over the past seven years. The wool is jetted with white spirit on a special conveyor followed by similar jetting with cold water. Solvent recovery is by centrifuging and vacuum

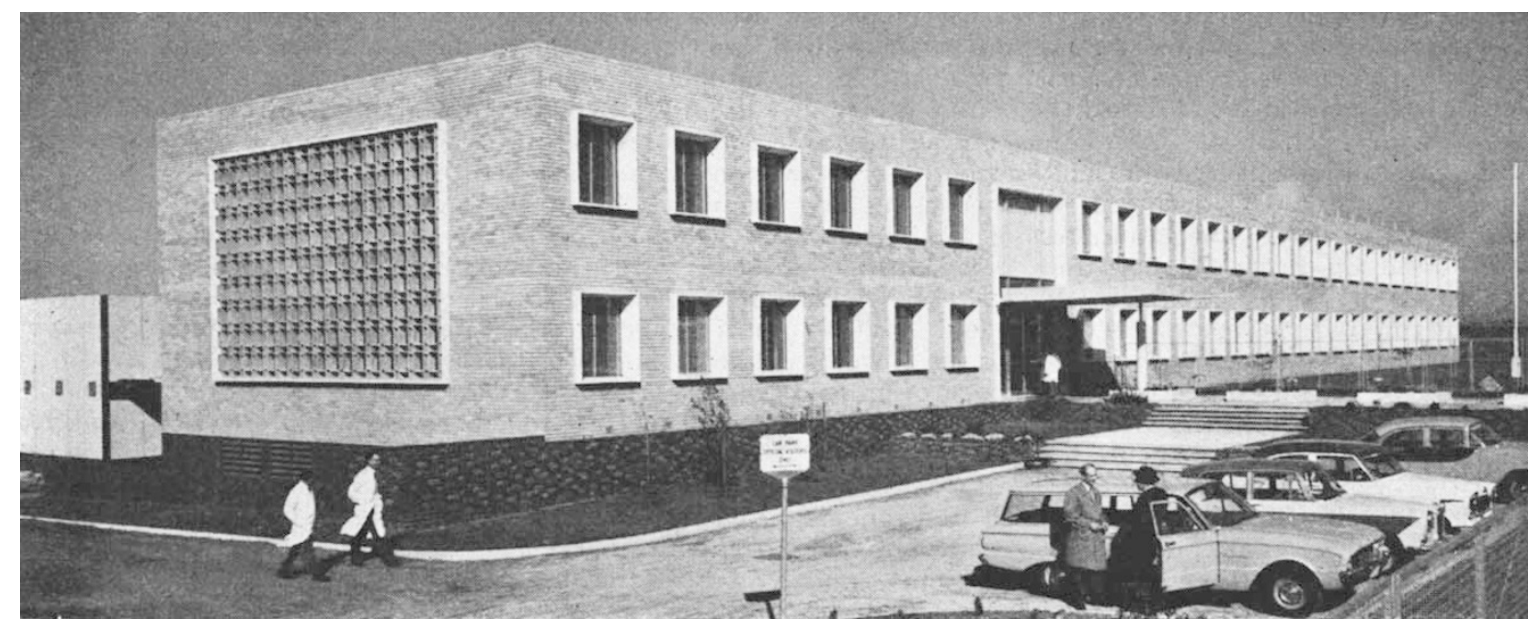

Fig. 1. New C.S.I.R.o. Wool Research Laboratory, Geelong, Victoria 
distillation. Extension of this technique to aqueous detergent jet scouring is also taking place. Both methods have been found to cause less felting than when the wool is scoured in the normal way. This leads to better processing performance with less fibre breakage in subsequent carding and combing. Lanolin recovery and utilization is also another activity. Methods of purifying and bleaching lanolin have been developed and utilization of various fractions is now being investigated.

Research on worsted earding has shown that addition of 0.5 per cent lubricant of about 30 centipoise viscosity greatly improves carding performance, reducing fibre breakage and waste. This was predicted from fibremetal frictional measurements and then confirmed in processing trials. Studies on friction are being extended along more fundamental lines investigating short-term high-temperature rises vccurring at contact points when fibres are rubbed. This is part of a general programme related to the drafting of fibres in worsted processing.

Apart from improving existing machines, research is also in progress on the possibility of elimination of some types of conventional equipment. In particular, there has been considerable work on developing an alternative to the worsted card, which causes large fibre losses and breakage when the wool passes through it. A machine known as the sliver converter has been developed for this purpose, of which an industrial prototype is at present under construction in the United States. The converter will occupy considerably less space than the card and should substantially reduce fibre breakage and loss.

The new laboratories will permit an extension of these activities and provide space for several additional projects.

\title{
KUMASI CONFERENCE ON THE LAKE BOSUMTWI CRATER
}

\section{By S. O. BAMPO}

\author{
Department of Crystallography, Birkbeck College, University of London
}

\begin{abstract}
$\Delta \mathrm{T}$ the fourth conference of the West African Science A Association, held at the Kwame Nkrumah University of Science and Technology, Kumasi, Ghana, during March 22-26, scientists from parts of West Africa, Europe, China and U.S.S.R. were represented. The conference this year had as a central theme the question of the origin of the Lake Bosumtwi crater. This was of particular interest owing to the recent controversy on the volcanic or meteoritic origin of isolated craters. The discovery of coesite in a specimen from the Bosumtwi site (1961) has led to the suggestion by Dietz, Chao, Litter and Fahey ${ }^{1}$ that the depression has a meteorite impact origin. This is in contradiction to the cryptovolcanic origin theory proposed by Junner ${ }^{2}$ in 1933.

Lake Bosumtwi is situated in the central part of Ashanti in Ghana and lies on lat. $6^{\circ} 30^{\prime} \mathrm{N}$. and long. $1^{\circ} 25^{\prime} \mathrm{W}$. It is fairly circular in shape with a diameter of about $6 \frac{1}{2}$ miles. The crater base stands at a depth of $1,150 \mathrm{ft}$. from the raised rim of the depression, which is carved out of lower Birrimian (Pre-Cambrian) rocks. No lava flows have been found in the intensive geological surveys made so far. However, small deposits of brecciated conglomerates and tuff have been discovered ${ }^{2}$ near Mmorontuo village, about $1 \frac{1}{2}$ miles from the crater, and in the Boni stream north-north-east of the crater. A correlation has been drawn between the analyses of some of these breccia and dacite from Scotland. An aerial magnetic survey of the lake district in 1962 has revealed a negative anomaly of amplitude 40 gammas striking in an east-west direction inside the crater.
\end{abstract}

Among the distinguished visitors to the conference was Prof. Gentner, director of the Max-Planck Institute for Nuclear Research, Heidelberg, who has for the past two years been working on the dating of tektites using the potassium-argon method. Large numbers of tektite finds have been dated by Profs. W. Gentner and Zahringer, including the Ivory Cosst tektites (Ouelletites), which have been estimated to be $1.3 \mathrm{~m}$. years old. These tektites have been associated by Cohen ${ }^{8}$ with the Bosumtwi depression based on the terrestrial impact origin of tektites. In connexion with the various groups of tektite ages, Prof. Gentner discussed the several hypotheses concerning the origin of tektites.

Prof. Th. Monod, the director of Institut Français d'Afrique Noire, University of Dakar, read a paper on craters and astroblemes-general problems and West African structures. In this group he included the Bosumtwi feature and the Aouelloul structure in Mauritania, which is considered most probably to be an astro- bleme. He has in preparation also a list of various astroblemes and their statistical data, which when completed may be a very valuable contribution to this type of investigation.

I, now on leave from the Kwame Nkrumah University of Science and Technology, read a paper on the Bosumtwi crater, in which the problem was examined in considerable detail.

In my paper, claims for a voleanic origin of the Bosumtwi issue were discussed. This was necessary because Maclaren in 1931 had proposed a meteorite impact theory which was dismissed at the time on account of the paucity of evidence of meteorite fragments and any other indication of meteorites. Apparently, the discovery of the deposits of Mmorontwo conglomerates (1932), including the analysis of a black, glassy material which looked like dacitic pitchstones from Scotland, seems to have strengthened the crypto-volcanic theory suggested by Junner.

I suggested that in the light of the present knowledge of such structures, it has been found that the absence of large deposits of volcanic material around such structures may not be strong enough evidence for all the structures to be attributed to volcanic origin. In addition, since Ghana lies outside the region of volcanic activity in Africa, a volcanic theory would need to be treated with caution. Secondly, the black dacite-like material examined appeared to contain about 3 per cent more water below $110^{\circ} \mathrm{C}$ than the Scottish dacites with which they have been compared. Differences in iron and magnesium contents of the specimens were also noticed. I discussed experiments on high-speed impact by Charter and Summers ${ }^{4}$, from which I drew a correlation between known meteorite crater and experimental crater depthdiameter curve. I suggested that the Bosumtwi crater, if it had been of impact origin, would most probably have been formed in the fluid impact stage; and that the accompanying high velocity and pressure on the projectile would have caused it after impact to behave more like a fluid than a solid body. From this I went on to discuss the theory of erater formation by the hydrodynamical approach, as suggested by Culp and Hoopes. This was based on the development of shock-wave fronts in the fluid resulting in the production of minerals of rare density and crystal structure under high pressures and temperatures. This was in agreement with similar analyses by Staninkovich and Fedynskii ${ }^{6}$, who postulated that when a meteorite hits the Earth with a velocity greater than $4 \mathrm{~km} / \mathrm{sec}$ it might explode after impact to produce a large crater. 